\title{
GOBERNANZA Y DERECHO DE AGUAS EN CHILE
}

\section{Governance and Water Law in Chile}

\author{
Nicolás Cannoni Mandujano* \\ JuAN José CROCCO CARRERA** \\ Universidad Autónoma de Chile \\ Santiago, Chile
}

RESUMEN: En este trabajo estudiamos los elementos de gobernanza presentes desde su origen en el Derecho de Aguas chileno, revisando en especial la regulación de las organizaciones de usuarios de aguas, en tiempos donde la conflictividad ha ido en aumento con ocasión del cambio climático, la condición de sequía y la sobreexplotación del recurso hídrico, siendo imperativo apuntar hacia una regulación que contemple sistemas de gestión, que concilie los distintos intereses que confluyen en torno a la utilización de las aguas en una misma cuenca u hoya hidrográfica. De acuerdo a lo expuesto en este trabajo, para que exista una buena gobernanza es necesario que se incorporen nuevos actores en la toma de decisiones en torno al manejo de las aguas.

PALABRAS CLAVE: Derecho de Aguas, gobernanza, organizaciones de usuarios

\footnotetext{
Licenciado en Derecho, Pontificia Universidad Católica de Chile. Máster en Ciencias, Universidad de Heidelberg, Alemania. Profesor de Derecho Regulatorio, Universidad Autónoma de Chile. Abogado en Aylwin, Mendoza, Luksic \& Valencia. Correo electrónico: $<$ ncannoni@amlv.cl>.

** Licenciado en Derecho, Pontificia Universidad Católica de Chile. Abogado en Crocco Bulnes. Correo electrónico: <jcrocco@croccobulnes.cl>.
}

Artículo recibido el 25 de diciembre de 2016 y aceptado para publicación el 30 de abril de 2017. 
ABSTRACT: This paper examines the elements of governance content in the Chilean Water Law since its origin, reviewing in particular the regulation of the water user associations, in times where the conflicts have been increasing because of the climate change, drought conditions and overexploitation of water resources, so it is imperative to look for a regulation that includes management systems that reconciles the various interests that converge around the use of water in a same basin or watershed. According to this paper, for getting good governance, it is required that new actors should be included in the decision-making around water management.

KEYWORDS: Water Law, governance, user associations

\section{INTRODUCCIÓN: SITUACIÓN ACTUAL EN RELACIÓN A LA GESTIÓN DE RECURSOS HÍDRICOS}

Actualmente los recursos hídricos enfrentan una crisis a nivel mundial, derivada principalmente de la condición de sequía provocada por el cambio climático y de la sobreexplotación del recurso hídrico, lo que se ha visto agravado por una crisis de gobernanza. ${ }^{1}$ Chile no es la excepción y ha enfrentado estos últimos años una grave situación de sequía, además de una creciente demanda por el uso de aguas, que ha provocado la sobreexplotación de las cuencas hidrográficas y acuíferos. ${ }^{2}$ Uno de los aspectos relevantes en todo sistema de aprovechamiento y gestión de las aguas es la normativa, que en Chile encuentra a su principal fuente en el Código de Aguas de 1981, cuerpo legal cuya reforma se está discutiendo actualmente en el Congreso Nacional. ${ }^{3}$

Es importante tener presente que el desarrollo económico en Chile encuentra un pilar fundamental en la utilización de recursos naturales, en especial el agua, toda vez que prácticamente todas las actividades económicas utilizan el recurso hídrico como un insumo esencial en sus procesos

"2॰ Informe sobre el desarrollo de los recursos hídricos en el mundo", Organización de las Naciones Unidas: Comunicado de prensa n 14-2006. Disponible en: <http://www. unep.org/Documents. Multilingual/Default.asp?DocumentID $=471 \&$ ArticleID $=5215 \& \mathrm{I}=$ en>, fecha de consulta: 21 de junio de 2015 y Organización PARA LA CoOperación y el DeSARrollo ECONÓMICOS (2011).

Cfr. Dirección General de Aguas y Ministerio de Obras Públicas (2014).

"Proyecto de Ley que Reforma el Código de Aguas". Boletín n 7543-12, 8 septiembre 2014. Disponible en: <https://www.camara.cl/pley/pley_detalle.aspx?prmID=7936>, fecha de consulta: 5 de mayo de 2017. 
productivos. ${ }^{4}$ En nuestro país, históricamente, la demanda por el recurso hídrico ha provenido principalmente de la agricultura, reflejo de ello es que el Código de Aguas de 1981 tiene una notoria vocación agrícola. Hoy en día, la agricultura sigue siendo una actividad del todo relevante para nuestra economía, no solo por la generación de empleos y por las relaciones que se tienen con los productores locales, sino también debido a la importancia que tiene Chile dentro del mercado agrícola mundial. No obstante lo anterior, en la actualidad el consumo de agua proveniente de la agricultura debe competir con otras actividades de la economía nacional, de igual relevancia, entre las que cabe mencionar la industria minera, el sector sanitario y la generación de energía eléctrica. Así, en ocasiones los intereses respecto del uso del recurso pueden ser contradictorios entre uno y otro sector, como por ejemplo, la necesidad de almacenar agua para su uso en la generación hidroeléctrica, es contrapuesto a la demanda por el uso agrícola de esas mismas aguas en el verano.

De esta forma, el agua, en su calidad de recurso común, presenta una serie de dificultades, desde el momento en que varios individuos conviven en una misma fuente, y por lo tanto, se hace imperativo apuntar hacia un sistema de gobernanza de las aguas, capaz de conciliar los diversos intereses que confluyen en una misma cuenca y necesidades que no pueden ser satisfechas íntegramente, de manera tal de dotarse de reglas que permitan una sana coexistencia entre los interesados, para así administrar este recurso variable y-algunas veces- impredecible.

En razón de esta situación de hecho, provocada por el uso común de unas mismas aguas, la solución chilena desde sus orígenes consistió en la creación de organizaciones de usuarios de aguas. Éstas, en un principio y por mucho tiempo no tuvieron un marco jurídico que las regulara, por lo que los interesados, a fin de solucionar los conflictos derivados de este uso comunitario, debieron remitirse a las normas del Derecho Civil. Así las cosas, no fue sino hasta varios años después que la necesidad de regularizar esta situación forjó una normativa propia. Esto ha sido una característica muy relevante de la legislación en materia de organizaciones de usuarios, ya que su evolución se ha visto marcada por reacciones a posteriori, según se analizará más adelante. A partir de lo expuesto y, considerando que Chile está en un punto de inflexión en materia de legislación de aguas, con reformas en trámite de por medio, pareciera ser un buen momento para revisar los elementos de gobernanza presentes en la regulación de este recurso natural, a fin de incorporar

$4 \quad$ Donoso et al. (2010) p. 5. 
nuevos elementos y mejorar los ya existentes, siendo ello el objetivo general de este trabajo.

En el presente documento, primeramente, se presenta el contexto en atención a los diversos usos que se da a las aguas en Chile. En seguida, se describe el marco teórico en el cual se enmarca este artículo, en el cual se trata el concepto de gobernanza en general, y de gobernanza del agua en particular. A continuación, se presenta la normativa de aguas que rige actualmente en nuestro país, tratándose los aspectos generales de esta regulación y la evolución que ha tenido en materia de organizaciones de usuarios, y luego se enuncian las modificaciones que pretenden introducirse al Código de Aguas vigente. Posteriormente, se presenta un análisis de los resultados a los que se llegó en el presente trabajo, identificando los elementos de gobernanza observados en el Derecho de Aguas chileno, para finalmente terminar con las conclusiones de rigor y el respectivo listado de las fuentes bibliográficas consultadas.

\section{CONTEXTO DE LA UTILIZACIÓN DE LOS DERECHOS DE APROVE- CHAMIENTO DE AGUAS}

Los derechos de aprovechamiento que se otorgan sobre las aguas terrestres pueden ser de uso consuntivo o de uso no consuntivo, siendo los primeros aquellos en que se puede consumir totalmente las aguas y en cualquier actividad, mientras que los segundos son aquellos en que el titular, luego de utilizar las aguas, debe restituirlas en igual cantidad, calidad y substancia. ${ }^{5}$ Los derechos de aprovechamiento que se utilizan en el desarrollo de la agricultura son esencialmente aquellos de uso consuntivo, mientras que el ejemplo clásico de utilización de derechos de aprovechamiento de uso no consuntivo es la generación hidroeléctrica.

En Chile, el sector agrícola es el principal usuario de derechos de aprovechamiento de uso consuntivo, con un $77,8 \%$, seguido muy de lejos por el

$5 \quad$ El artículo $13^{\circ}$ del Código de Aguas de 1981, establece que: "Derecho de aprovechamiento consuntivo es aquel que faculta a su titular para consumir totalmente las aguas en cualquier actividad". Mientras que, por otro lado, el artículo $14^{\circ}$ del mismo cuerpo legal prescribe que: "Derecho de aprovechamiento no consuntivo es aquel que permite emplear el agua sin consumirla y obliga a restituirla en la forma que lo determine el acto de adquisición o de constitución del derecho. La extracción o restitución de las aguas se hará siempre en forma que no perjudique los derechos de terceros constituidos sobre las mismas aguas, en cuanto a su cantidad, calidad, substancia, oportunidad de uso y demás particularidades". 
sector industrial $(9,1 \%)$, la industria minera $(7,2 \%)$ y la producción de agua potable $(5,9 \%)$. Observando conjuntamente los usos consuntivos y no consuntivos, clasificados según recaigan sobre aguas superficiales o subterráneas, se constata la predominancia del uso agrícola en los usos consuntivos, mientras que en los no consuntivos lideran el uso las actividades industriales, piscicultura y generación de hidroelectricidad. ${ }^{6}$ Sin perjuicio de lo antes indicado, cabe tener presente que muchas de las aguas utilizadas en las faenas mineras corresponde a aquellas denominadas "aguas del minero", que son aquellas aguas a las que la minería tiene acceso por el solo ministerio de la ley sin la necesidad de contar con un título otorgado por la autoridad. ${ }^{7}$ La disponibilidad de aguas en Chile enfrenta una situación compleja. En los últimos años se ha declarado zona de escasez hídrica en diversas cuencas del norte, debido a la incesante sequía. Asimismo, en el centro-sur cada año se decreta emergencia agrícola e hídrica debido a la sequía estival.

Los efectos del cambio climático han impactado en los recursos hídricos, acrecentando una condición de sequía, lo que repercute negativamente, por ejemplo, en la agricultura. En efecto, Nelson y otros autores plantean que: "La agricultura es extremadamente vulnerable al cambio climático. El aumento de las temperaturas termina por reducir la producción de los cultivos deseados, a la vez que provoca la proliferación de malas hierbas y pestes. Los cambios en los regímenes de lluvias aumentan las probabilidades de fracaso de las cosechas a corto plazo y de reducción de la producción a largo plazo. Aunque algunos cultivos en ciertas regiones del mundo puedan beneficiarse, en general se espera que los impactos del cambio climático sean negativos para la agricultura, amenazando la seguridad alimentaria mundial" ${ }^{8}$ Agregan los mismos, ${ }^{9}$ que el cambio climático produce una alteración significativa

$6 \quad$ VALDÉs-PINEDA et al. (2014) p. 2549.

$7 \quad \mathrm{Al}$ respecto, las denominadas "aguas del minero" están reguladas en el inciso final del artículo 56 del Código de Aguas de 1981 que establece lo siguiente: "Corresponde a los dueños de pertenencias mineras, dentro de ellas, el derecho de aprovechamiento de las aguas halladas en sus labores, mientras conserven el dominio de sus pertenencias y en la medida necesaria para la respectiva explotación". Este artículo está complementado a su vez por el artículo 110 del Código de Minería de 1983, que prescribe que: "El titular de concesión minera tiene, por el solo ministerio de la ley, el derecho de aprovechamiento de las aguas halladas en las labores de su concesión, en la medida en que tales aguas sean necesarias para los trabajos de exploración, de explotación y de beneficio que pueda realizar, según la especie de concesión de que se trate. Estos derechos son inseparables de la concesión minera y se extinguirán con ésta."

$8 \quad$ Nelson et al. (2009) p. VII.

9 Ídem., p. 4. 
en las posibilidades de riego, toda vez que las precipitaciones disminuyen y el agua renovable es aquella que proviene de las lluvias, y que -ademáshay que considerar que el aumento de temperaturas, inducido por el cambio climático, causa un incremento de los requerimientos hídricos de los cultivos, y la relación entre el consumo de agua y la necesidad de los cultivos se denomina confiabilidad del abastecimiento de aguas de riego.

Saldívar ${ }^{10}$, por otra parte, indica que el factor cambio climático provoca una mayor complejidad y vulnerabilidad al problema de los recursos hídricos, su localización, escasez y manejo. En tanto, Valdés-Pineda et al. ${ }^{11}$ señalan que el cambio climático genera una alta incertidumbre hídrica en los escenarios futuros, por lo que en este contexto el futuro se ve poco auspicioso desde una perspectiva de disponibilidad de aguas. A mayor abundamiento, esto acarrea aumentos adicionales en los precios de los principales cultivos, tales como el arroz, trigo, maíz y soja. ${ }^{12}$

Como ya se ha señalado, la crisis del agua no solo se produce por una baja en la disponibilidad del recurso en sus fuentes naturales, sino que también por el aumento exponencial en la demanda por el mismo. De esta forma, la necesidad de declarar zona de escasez hídrica y/o emergencia agrícola, si bien responde a una baja en la disponibilidad, es una medida excepcional tendiente a establecer o redefinir las reglas de distribución entre los usuarios de las aguas, con el objeto de hacer frente a la crisis y conciliar los diversos intereses que existen respecto del uso del recurso hídrico. En suma, aun cuando podamos tomar medidas de mitigación respecto del cambio climático, los efectos de las mismas pueden tardar muchos años en evidenciarse, por lo que mientras no seamos capaces de controlar la variable disponibilidad del recurso, debemos enfocar todos nuestros esfuerzos en la variable demanda, potenciando y fortaleciendo las instituciones destinadas a la gobernanza del agua.

\section{GOBERNANZA DE LAS AGUAS}

\section{La gobernanza: análisis conceptual y evolución del término}

Como se adelantó, el presente trabajo se enmarca dentro de la gobernanza del agua. Antes de adentrarnos en ese concepto, cabe señalar que el concepto de gobernanza, en general, tradicionalmente se asemejaba a gobierno,

\footnotetext{
$10 \quad$ SAldívar (2013) p. 75.

11 Valdés-Pineda et al. (2014) p. 2563.

12 Nelson et al. (2009) p. 6.
} 
teniéndoseles como sinónimos. ${ }^{13}$ Sin embargo, el término "gobernanza" ha evolucionado a diferentes acepciones como, por ejemplo: "para indicar un nuevo estilo de gobierno, distinto del modelo del control jerárquico y caracterizado por un mayor grado de cooperación y por la interacción entre el Estado y los actores no estatales al interior de redes decisionales mixtas entre lo público y lo privado", o entendiéndola como "una modalidad distinta de coordinación de las acciones individuales, entendidas como formas primarias de construcción del orden social". ${ }^{14}$

Entonces, la gobernanza ha avanzado conceptualmente hacia la incorporación de otros actores de la sociedad en la toma de decisiones, surgiendo así como una alternativa a la mera conducción política jerárquica tradicional. En esto ha tenido injerencia la necesidad de legitimar las decisiones que se adoptan. En este sentido, Stoker ${ }^{15}$ plantea que, para que las decisiones sean legítimas, se requieren básicamente tres condiciones: (i) la conformidad de la decisión con las normas establecidas; (ii) su justificación en las creencias compartidas por la sociedad; $y$, (iii) el consentimiento expreso de los subordinados, o de los más significativos entre ellos.

De tal manera, para Rosas-Ferrusca" ${ }^{16}$ la gobernanza "significa que ha nacido una sociedad gubernamentalmente independiente, autónoma y competente, poseedora de capacidades que el gobierno no posee y que requiere para conducir a la sociedad; por lo que la dirección debe ser una actividad compartida y asociada entre gobierno y sociedad, en una relación de interdependencia más que de dependencia y de coordinación más que de subordinación", y agregan que "en síntesis, la gobernanza es el proceso en el que los ciudadanos resuelven colectivamente sus problemas y responden a las necesidades de la sociedad, empleando al gobierno como instrumento para llevar a cabo su tarea". Por su parte, Nanda ${ }^{17}$ señala que para el éxito de la gobernanza, el Estado y las instituciones deben ser reformados y fortalecidos; las instituciones deben ser democráticas; y ha de promoverse una efectiva participación y establecerse responsabilidades, además de intensificarse el rol de la ley como herramienta para asegura una buena gobernanza y que ésta sea sostenible.

\footnotetext{
13 StOKer (1998) p. 17 y MAYNTz (1998) p. 1.

Así, MAYntz (1998) p. 1, señala que el término gobernanza (governance) se asimilaba a Politische Steuerung (dirección política).

14 Ibídem.

15 STOKER (1998) p. 21.

16 Rosas-Ferrusca et al. (2012) pp. 118 y 119.

$17 \quad$ Nanda (2006) pp. 280 y 281.
} 
A pesar de estas concepciones, para Ruiz y Gentes el término "gobernanza" es un concepto amplio, lo que se aprecia según estos autores al citar a Tegegnework Gettu, entonces Directora Ejecutiva de la Oficina del Programa de Naciones Unidas para el Desarrollo (PNUD), que señaló que: "La gobernanza es un concepto que comprende mecanismos complejos, procesos, relaciones e instituciones a través del cual los ciudadanos y grupos articulados por sus intereses, ejercen sus derechos y obligaciones, y median sus diferencias. El buen gobierno dirige la gestión de la asignación de recursos para responder a los problemas colectivos, se caracteriza por los principios de participación, transparencia, responsabilidad, reglamentos de ley, efectividad, equidad y visión estratégica. Es un concepto universal importante y aún complejo".$^{18}$ Es decir, de los conceptos expuestos, consta que el término gobernanza ha evolucionado desde entenderlo como sinónimo de gobierno, hasta hoy, que introduce a la interacción social como elemento esencial para la validación y legitimidad en la toma de decisiones. Sin embargo, para los mismos, la gobernanza "no implica anular o subestimar las atribuciones del gobierno, sino propone más bien un cambio en el ejercicio de la administración pública tendiente a la coordinación y organización de un espacio deliberativo, basado en la confianza, la participación y el control social". ${ }^{19}$

De esta manera, ha de existir un equilibrio entre la interacción de agentes públicos y privados. Así las cosas, en términos simples, para los efectos de esta investigación, la gobernanza es un modelo de gestión pública que contempla procesos formales e informales en la toma de decisiones, que involucra a los actores públicos, sociales y privados, con intereses similares u opuestos. $^{20}$

\section{Gobernanza del agua y sus elementos}

Vista la conceptualización de la gobernanza en general, es preciso ahora referirse en particular a la gobernanza del agua. La gobernanza del agua se está convirtiendo en un área de estudio de creciente importancia para los hidrólogos, por cuanto se reconoce el impacto que el manejo de los cauces tiene en las decisiones humanas en diferentes niveles. La gobernanza del agua influye en el "sistema social-hidrológico" y así afecta, como también es afectada, por las decisiones que se toman en relación a variadas áreas, como

\footnotetext{
18 Ruiz y Gentes (2008) p. 55.

19 Ídem., p. 43.

20 Ídem., pp. 42 y 43.
} 
la selección de cultivos, políticas de uso de suelo, energía y gestión del medio ambiente. ${ }^{21}$

Para la Organización para la Cooperación y el Desarrollo Económicos $(\mathrm{OECD})^{22}$ la gobernanza del agua corresponde al conjunto de reglas, prácticas y procesos en los cuales se toman e implementan las decisiones para el manejo del recurso hídrico y los servicios, y donde los tomadores de decisiones son responsables y deben rendir cuentas. Así, en la actualidad hay una necesidad urgente de hacer un balance de las experiencias recientes, identificar las buenas prácticas y desarrollar herramientas para ayudar, en los distintos niveles, ya sea gubernamentales o de otras partes interesadas, en la participación de políticas relacionadas con el agua que sean eficaces, justas y sostenibles. La gobernanza del agua comprende entonces los mecanismos, procesos e instituciones a través de los cuales el Estado y la sociedad civil articulan sus intereses y solucionan sus diferencias, de manera tal de satisfacer las necesidades de consumo de los diferentes sectores y así proteger el recurso hídrico, emergiendo como la capacidad social de movilizar energías para el desarrollo sustentable.

Algunas condiciones identificadas por Ruiz y Gentes para la gobernanza del agua son "la sociedad civil articulan sus intereses y solucionan sus diferencias, de manera tal de satisfacer la participación, para la conservación del recurso; multiplicar el valor de la transparencia y comunicación en la red de actores; y establecer un régimen regulatorio que incluya equitativamente las diversas necesidades e intereses sociales". ${ }^{23}$ Uno de los principios (Principio 1) para la gobernanza del agua establecido por la $\mathrm{OECD}^{24}$ es ubicar y distinguir, claramente, las responsabilidades, multiplicar el valor de la transparencia y comunicación en la red de actores; y establecer un régimen de regulación entre las distintas autoridades responsables. Para estos efectos, resulta fundamental un marco legal e institucional que se haga cargo de ello.

Así, se plantea que la regulación debe establecer autorizaciones, monitoreos, fiscalizaciones y manejo de conflictos, entre otras cosas, además de identificar las lagunas y conflictos de intereses a nivel gubernamental. En seguida, otro principio (Principio 7) que señala la OECD ${ }^{25}$ apunta a asegurar que los marcos regulatorios relacionados con el manejo del agua sean

\footnotetext{
21 Daniell y Barreteau (2014) p. 2367.

22 Organización Para la Cooperación Y El Desarrollo Económicos (2015) p. 6.

23 Ruiz Y Gentes (2008) p. 43.

24 Organización Para la Cooperación Y El Desarrollo Económicos (2015) p. 10.

25 Ídem., p. 12.
} 
efectivamente implementados y tiendan a conseguir y resguardar intereses públicos. En ese sentido, se plantea la necesidad de reglas, instituciones y procesos que sean bien coordinados, transparentes, no discriminatorios, participativos y fáciles de entender y de hacer cumplir, fomentando herramientas regulatorias eficientes y asegurando el acceso a la justicia. Así, la gobernanza del agua tiene una dimensión administrativa o legal. En este aspecto hay distintos niveles de organizaciones a nivel local, provincial, regional, nacional y supranacional y, generalmente, ejercen jurisdicción y competencias de acuerdo a la regulación en un área geográfica específica. ${ }^{26}$

Por lo tanto, se puede observar que un elemento importante de toda gobernanza es la regulación. Así, el papel de la legislación de aguas en la gobernanza del recurso hídrico es preponderante, la cual ha de incorporar la interacción con los diferentes actores de la sociedad que serán los receptores de tal marco regulatorio. Como se señaló anteriormente, tradicionalmente la gobernanza era entendida como sinónimo de gobierno y, según Mayntz, el primer paradigma de la teoría de la governance "tenía, pues, relación con los temas del desarrollo e implementación de las políticas públicas, y adoptaron lo que podemos definir como una perspectiva desde arriba (top-down), es decir, desde el punto de vista del legislador".$^{27}$ Pero, agrega ${ }^{28}$ que lo que no se consideraba en ese entonces era la resistencia obstinada por parte de los destinatarios de dichas políticas, así como su capacidad para obstaculizar el logro de los objetivos pretendidos por la misma, por lo tanto, esa perspectiva top-down ha de evolucionar hacia una interacción entre los distintos actores, precisamente para evitar esa obstaculización.

En estos momentos, como se ha expuesto, Chile está en proceso de cambios legislativos en relación a las aguas, existiendo actualmente un cuerpo legal dictado en 1981 bajo un paradigma neoliberal que se intenta reformar, hacia una normativa que, esencialmente, privilegie y asegure el consumo humano del agua como derecho fundamental, y es indudable la importancia que adquiere que se incorporen elementos de gobernanza en esta modificación en trámite. En consistencia con lo señalado precedentemente, para Ruiz y Gentes la gobernanza "resulta de la creación y gestión de redes o estructuras de relación que involucran a diferentes tipos de actores, cuya interacción es crucial para enfrentar los desafíos más urgentes" ${ }^{\prime 29}$. Asimismo, indican que la gobernanza hídrica comprende un conjunto de medidas

\footnotetext{
26 Daniell y Barreteau (2014) p. 2368.

$27 \quad$ MAYNTZ (1998) p. 2.

28 Ídem., p. 3.

29 Ruiz y Gentes (2008) pp. 43, 53 y 54.
} 
que van más allá de la aprobación de $\operatorname{leyes}^{30}$ o la creación de instancias nacionales, sino que también está condicionada por el ejercicio equitativo de los derechos y deberes sobre los recursos por parte de todos los actores sociales y la consideración de los diferentes intereses y poderes sectoriales de los usuarios del agua. En este orden de ideas, aparecen como elementos esenciales de la gobernanza, en este caso aplicada a las aguas: (i) la capacidad de movilizar energías articulando intereses; y, (ii) la importancia de que las políticas públicas sean socialmente aceptadas.

Valdés-Pineda et al. ${ }^{31}$, plantean que para una buena gobernanza futura, Chile requiere, entre otras cosas, modificar la institucionalidad; crear políticas que fomenten la gestión integrada de recursos hídricos, que contemple el manejo de los recursos superficiales y subterráneos en forma integral; mejorar el uso eficiente del agua incentivando la inversión privada hacia nuevas tecnologías e invertir en infraestructura relativa a acueductos, canales, recarga de acuíferos, desalinización y medidores; definir nuevos instrumentos legales para contribuir a la descontaminación de las fuentes de aguas para evitar su degradación; mejorar la calidad y cantidad de los instrumentos y elementos técnicos para una mejor comprensión y nivel de conocimiento de los recursos hídricos superficiales y subterráneos, para así permitir un apropiado manejo y propiciar políticas y decisiones adecuadas; aumentar las fuentes de financiamiento para investigaciones relacionadas con el agua, la infraestructura y proyectos de administración; promover una cultura orientada a la conservación de los recursos hídricos, realizando campañas comunitarias, programas escolares y eventos en general, entre otros, y, por último, de total relevancia para estos efectos, fortalecer las organizaciones de usuarios, creando políticas que favorezcan su creación y registro.

Así las cosas, la gobernanza del agua en Chile está en un punto de inflexión, el cual puede conducir a un cambio de régimen institucional del agua (por ejemplo, reformar el Código de Aguas) o mantener el status quo. Que vaya en una o en otra dirección, según señala Retamal, "dependerá si los sistemas políticos, sociales, económicos y administrativos comparten el poder a la hora de tomar decisiones en la gestión del agua y si se coordinan e integran para que la gobernanza del agua proceda hacia una trayectoria de sustentabilidad". ${ }^{32}$

\footnotetext{
30 Ruiz y Gentes (2008) p. 52, señalan que el empoderamiento local no se aplica por ley, sino que por un gran pacto o acuerdo social.

31 VALDÉs-PINEDA et al. (2014) p. 2563.

32 Retamal et al. (2013) p. 14.
} 
A partir de lo señalado y conceptos indicados, para los efectos de este trabajo, la gobernanza del agua contiene los elementos que se identifican a continuación: i) interacción Estado y actores no estatales (proceso participativo) en la toma de decisiones, es decir, se constata una actividad compartida y asociada entre gobierno y sociedad (redes decisionales mixtas entre lo público y lo privado), donde existe una relación de interdependencia más que de dependencia; ii) necesidad de lograr un mecanismo de manejo y gestión del agua que satisfaga las necesidades de consumo de los diferentes sectores y así mismo proteger el recurso natural con apoyo de todos los actores involucrados en su manejo; iii) capacidad de diseño de políticas públicas que sean socialmente aceptadas, evitando así la resistencia obstinada por parte de los destinatarios de dichas políticas, así como su capacidad para obstaculizar el logro de los objetivos pretendidos por las mismas; $y$, iv) dimensión legal (nivel local, provincial, regional, nacional, supranacional) que asegura que los marcos regulatorios relacionados con el manejo del agua sean efectivamente implementados por los actores involucrados y tiendan a conseguir y resguardar intereses públicos.

El próximo apartado se referirá a la legislación de aguas en Chile, en especial al Código de Aguas de 1981, actualmente vigente, en sus aspectos generales y en lo relativo a las organizaciones de usuarios, y, además, se resumen los principales lineamientos de la reforma que se ha presentado para reformarlo. ${ }^{33}$

\section{DERECHO DE AGUAS EN CHILE Y SU VINCULACIÓN CON LOS USUA- RIOS}

\section{Aspectos generales del marco regulatorio chileno en materia de aguas}

Como se señaló precedentemente, el marco regulatorio es un aspecto relevante en la gobernanza del agua. En nuestro ordenamiento las aguas se consideran un bien nacional de uso público ${ }^{34}$, y su uso está sujeto a que quien

utiliza el recurso hídrico sea titular de un derecho de aprovechamiento de

33 "Proyecto de Ley que Reforma el Código de Aguas". Boletín n 7543-12, 8 septiembre 2014. Disponible en: <https://www.camara.cl/pley/pley_detalle.aspx?prmID=7936>, fecha de consulta: 5 de mayo de 2017.

34 El artículo $5^{\circ}$ del Código de Aguas de 1981, establece que: "Las aguas son bienes nacionales de uso público y se otorga a los particulares el derecho de aprovechamiento de ellas, en conformidad a las disposiciones del presente código". 
aguas. ${ }^{35}$ El derecho de aprovechamiento de aguas y su ejercicio está regulado esencialmente en el Código de Aguas dictado en 1981, y la propiedad de los particulares sobre los derechos de aprovechamiento está garantizada por la Constitución Política de la República (artículo 19, $\mathrm{n}^{\circ}$ 24, inciso final ${ }^{36}$ ). Es así como esta dualidad -bien público y bien privado-, que si bien jurídicamente recaen sobre cosas distintas (uno sobre el agua y el otro sobre el derecho de aprovechamiento), en la realidad física -el agua- se confunden.

El modelo consagrado por el Código de Aguas de 1981, consiste básicamente en un sistema de otorgamiento de derechos de aprovechamiento a título gratuito y perpetuo, en la medida que exista disponibilidad del recurso y que no se perjudiquen derechos de aprovechamiento de terceros existentes con anterioridad. Este modelo instaurado por el Código de Aguas de 1981 responde a que, como señala Mayntz, "en los primeros años de la década de los ochenta, los principios del mercado fueron examinados mayormente como la alternativa más eficaz respecto a la regulación de las políticas ambientales de los Estados Unidos. En aquel período, tales principios se convirtieron en la columna de la ideología política del neoliberalismo y del thatcherismo, promoviendo la desregulación y la privatización como medios para estimular el crecimiento y para incrementar la eficiencia económica". ${ }^{37}$ Así, este modelo fortaleció la calidad de bien productivo de las aguas, entendido como un bien económico necesario para el desarrollo del país y que, en consecuencia, tiene el mismo tratamiento que el resto de los bienes.

A más de diez años de su entrada en vigencia, el Código de Aguas de 1981 fue objeto de una reforma en el año 2005, introducida por medio de la Ley $\mathrm{n}^{\circ} 20.017$, donde una de las principales innovaciones fue el establecimiento de una patente por no uso, que han de pagar aquellos titulares de derechos de aprovechamiento que no los estuvieran utilizando. La implementación de esta patente tuvo por objeto frenar la especulación de quienes solicitaban derechos de aprovechamiento meramente para incorporarlos en su patrimonio, sin haber un uso efectivo asociado ni interés en hacerlo. También

35 Artículo $6^{\circ}$ del Código de Aguas de 1981. En tanto, los incisos $1^{\circ}$ y $2^{\circ}$ de este artículo, establecen que: "El derecho de aprovechamiento es un derecho real que recae sobre las aguas y consiste en el uso y goce de ellas, con los requisitos y en conformidad a las reglas que prescribe este Código. El derecho de aprovechamiento sobre las aguas es de dominio de su titular, quien podrá usar, gozar y disponer de él en conformidad a la ley".

36 El inciso final del artículo $19 \mathrm{n}^{\circ} 24$ de la Constitución Política de la República de Chile de 1980, establece que: "Los derechos de los particulares sobre las aguas, reconocidos o constituidos en conformidad a la ley, otorgarán a sus titulares la propiedad sobre ellos".

37 MAYNTZ (1998) p. 3. 
se estableció un caudal ecológico mínimo (artículo 129 bis ${ }^{38}$ ) que tiene como fin la preservación de la naturaleza y la protección del medio ambiente. ${ }^{39}$ Sin embargo, esta reforma introducida en el año 2005 distó mucho de ser un cambio importante, no obstante haber prometido cambiar totalmente el ordenamiento en materia de aguas. ${ }^{40}$

A continuación, nos referiremos en particular a la evolución normativa y situación particular de las organizaciones de usuarios en la regulación actual, dado que constituyen las entidades Ilamadas a gestionar la administración de las aguas a nivel local, incorporando titulares de derechos de aprovechamiento con diversos intereses y finalidades, las que debieran tener una regulación sólida que les permita realizar sus funciones y atribuciones en un marco de gobernanza.

\section{Evolución normativa de las organizaciones de usuarios}

Tal como se adelantó, las organizaciones de usuarios son entidades preocupadas de la administración de los recursos hídricos a nivel local. Pero, su regulación y orientación ha sido determinada por el riego, omitiéndose otros intereses que también confluyen en la gestión de los recursos hídricos. Por ello, es del caso analizar en esta parte la evolución normativa de las organizaciones de usuarios desde su origen hasta la situación actual.

Desde principios de la Colonia hasta la entrada en vigencia de la Ley $n^{\circ} 2.139$, de 1908, no existían organizaciones jurídicas preocupadas de la administración de las aguas, sino que los usuarios se agrupaban en entidades de hecho a las que eran aplicables -sin ser suficientes- las normas del cuasicontrato de comunidad del Código Civil, vigente a esa época ${ }^{41}$, y solo

38 El inciso primero del artículo 129 bis 1 del Código de Aguas de 1981, establece que: "Al constituir los derechos de aprovechamiento de aguas, la Dirección General de Aguas velará por la preservación de la naturaleza y la protección del medio ambiente, debiendo para ello establecer un caudal ecológico mínimo, el cual solo afectará a los nuevos derechos que se constituyan, para lo cual deberá considerar también las condiciones naturales pertinentes para cada fuente superficial".

39 Valdés-Pineda et al. (2014) p. 2550.

40 Segura (2009) pp. 1 y 2.

41 El artículo 836 del entonces vigente Código Civil establecía que "el uso de las aguas que corren por entre dos heredades corresponde en común a los dos riberanos, con las mismas limitaciones, i será reglado en caso de disputa por la autoridad competente, tomándose en consideración los derechos adquiridos por prescripción u otro título, como en el caso del artículo precedente, núm. $1^{\circ \prime \prime}$. 
algunos canales contaban con estatutos especiales. En efecto, Venegas señalaba que "pocos son los canales que, como el de Maipo, se rijen [sic] bajo ciertos estatutos especiales; en la mayor parte de los canales no importa regla alguna para su uso, como quiera que el párrafo del Código Civil que trata del cuasi contrato de comunidad se limita a consignar preceptos jenerales [sic] que no pueden aplicarse a las diversas situaciones que presenta el derecho de usar unas mismas aguas numerosos predios de reducida extensión" ${ }^{42}$ Posteriormente, en tanto, comenzaron a aparecer Ordenanzas que regulaban la administración de algunos cauces, a saber: Valle de Copiapó, Sociedad de Maipo, río Aconcagua y río Teno, todas de 1872, Huasco, de 1880, y Tinguiririca, de 1887.

Mención aparte merece la Ordenanza de enero de 1872 sobre distribución de las aguas en los ríos que dividen provincias y departamentos que, en suma, consagró, en su artículo 14, un principio de participación donde los mismos usuarios debían ponerse de acuerdo en la cantidad de agua que debía asignarse a cada uno como dotación, sin perjuicio de lo señalado por $\mathrm{Obando}^{43}$, en el sentido que cualquiera que tuviera interés por un adecuado reparto podía solicitar al juez competente que citara a un comparendo de usuarios del río o canal, para que en él se designara una autoridad encargada de hacer la distribución bajo la mirada de una junta de vigilancia. Esta Ordenanza fue derogada casi en su totalidad por el Título $11^{\circ}$ del Libro III del Código de Procedimiento Civil dictado en 1902, que trataba acerca de los juicios sobre distribución de aguas, en los cuales se hace referencia a los comuneros y a las juntas de vigilancia. ${ }^{44}$

Luego, en 1908 se dicta la Ley $\mathrm{n}^{\circ} 2.139$ sobre asociaciones de canalistas, que tuvo como principal objetivo reglamentar la organización y funcionamiento de las comunidades existentes y las que se formaran con posterioridad. Así, el artículo $1^{\circ}$ de dicha ley establecía que "[s]erán personas jurídicas i se regirán por las disposiciones de esta lei, las asociaciones formadas por los dueños de canales, que se constituyan en conformidad al artículo 20, con el objeto de tomar el agua de la corriente matriz, repartirla entre los accionistas i conservar i mejorar los acueductos". En otras palabras, junto con otorgarle personalidad jurídica a este tipo de organizaciones, se orientaba en la

\footnotetext{
$42 \quad$ VeneGas (1887) p. 102.

43 Obando (2009) p. 114.

44 En este Código de Procedimiento Civil, modificado a su vez en 1918, se estableció (artículo $828 n^{\circ}$ 6) la posibilidad de nombramiento de una junta de vigilancia o de un delegado para hacer efectivos los acuerdos o resoluciones adoptadas por los usuarios.
} 
distribución de las aguas a que tenían derecho sus miembros y la mantención y conservación de la obra común.

Posteriormente, comienzan los primeros intentos de codificación del Derecho de Aguas chileno. En 1927, el diputado Rafael Moreno Echeverría presenta un proyecto que, aunque fallido, consideraba a las organizaciones de regantes y juntas de vigilancia. Luego, en el mensaje presidencial de fecha 10 de junio de 1936, mediante el cual se presentó el proyecto que creaba un Código de Aguas, se señaló que: "Los fines de interés común de los regantes, que inspiraron al legislador para dictar la Ley sobre Asociaciones de Canalistas, se mantienen en el proyecto ampliados a situaciones semejantes y completados con preceptos que se hacían indispensables para tener una legislación armónica sobre el goce de las aguas comunes. Tres son los organismos que regulan este orden de cosas: las comunidades de aguas, las asociaciones de canalistas y las juntas de vigilancia". ${ }^{45}$ El mismo mensaje agregaba que: "Las Juntas de Vigilancia que hoy son producto de estipulaciones contractuales de poca eficacia y con atribuciones para un río determinado, pasan a ser instituciones de Derecho con jurisdicción efectiva sobre toda una cuenca para dirigir los intereses comunes de todas las asociaciones de canalistas, comunidades de aguas y beneficiarios de la hoya, para evitar los enojosos pleitos entre riberanos y para ser representantes, por decirlo así, de una región agrícola servida por un mismo sistema fluvial. El funcionamiento de las Juntas de Vigilancia se rige por las reglas dadas para las asociaciones de canalistas, con las modificaciones que su naturaleza y fines requieren". ${ }^{46}$ El referido proyecto presentado en 1936 fue materia de un oficio que el Presidente de la República envió a la Cámara de Diputados con el objeto que fuera retirado, debido a la intervención de diversas instituciones con intereses en el marco normativo de las aguas. Sin embargo, y debido a la moción presentada por varios diputados en la misma sesión, el proyecto fue reingresado, continuando así su tramitación, promulgándose finalmente la Ley $\mathrm{n}^{\circ} 8.944$, publicada en el Diario Oficial de fecha 11 de febrero de 1948, sin embargo, lo que se suponía que sería el primer Código de Aguas chileno, culminó con sucesivas prórrogas de vacancia legal, por lo que no entró en vigor.

Así las cosas, el 4 de enero de 1949 se presentó un nuevo proyecto de ley sobre el Código de Aguas, que se tradujo en la dictación de la Ley $\mathrm{n}^{\circ}$ 9.909, publicada en el Diario Oficial de fecha 28 de mayo de 1951 (denominado comúnmente como el Código de Aguas de 1951). De esta manera, desde los primeros intentos de codificación, las organizaciones de usuarios

45 Cámara De Diputados (1936) p. 666.

$46 \quad$ Ídem., pp. 666 y 667. 
se consideraron como una solución al uso común de las aguas por parte de los regantes.

Luego en el tiempo, nos encontramos con el denominado Código de Aguas de 1969, que no es sino el Código de Aguas de 1951 con las modificaciones introducidas por la Ley no 16.640 de 1967, de Reforma Agraria. En cuanto a la administración de las aguas, un problema que debieron enfrentar los regantes fue que al no haberse definido previamente los sistemas de riego en los predios expropiados, éstos mantenían las redes internas de canales, concedidos para satisfacer necesidades globales de cultivos programados por un solo propietario. Al subdividirse las propiedades, sucedió con frecuencia que dicha red no se ajustaba a los nuevos requerimientos, necesitándose modificaciones sustanciales en los canales y obras de arte, diseñadas para otras circunstancias.

A partir de 1973, y de forma coherente con los cambios políticos que acaecieron en nuestro país, se iniciaron una serie de cambios legislativos, entre los que se incluyeron las aguas. En este sentido, cabe señalar lo establecido en el artículo $1 \mathrm{n}^{\circ} 16$ del Acta Constitucional $N^{\circ} 3$ dictada en virtud del Decreto Ley no 1552 de 1976, que modificó la Constitución Política de 1925, el cual en su inciso final disponía que: "Un estatuto especial regulará todo lo concerniente al [...] dominio de aguas". En virtud de dicha disposición, con fecha 23 de abril de 1979, mediante el Decreto Ley $\mathrm{n}^{\circ} 2.603$, se modificó y complementó lo dispuesto en el Acta Constitucional № 3, agregando antes del inciso final del artículo $1 \mathrm{~N}^{\circ} 16$ un nuevo inciso que dispuso: "Los derechos de los particulares sobre las aguas, reconocidos o constituidos en conformidad a la Ley, otorgarán a sus titulares la propiedad sobre ellos". En este artículo se evidencia la forma en que ha sido construida la legislación de aguas chilena, al hacer mención a los derechos reconocidos, refiriéndose aquellos en los que no obstante no existir título o acto de autoridad alguno a su respecto, son igualmente protegidos y reconocidos, denominados derechos consuetudinarios. ${ }^{47}$

Por otra parte, en esta modificación se facultó al Presidente de la República para que: "dicte las normas necesarias para el establecimiento del Régimen General de las Aguas, que modifique o reemplace, total o parcialmente, el Código de Aguas y las demás normas relativas a la misma materia". Luego de sucesivas prórrogas, finalmente, el 29 de octubre de 1981 es publicado el Decreto con Fuerza de Ley $n^{\circ} 1.122$, que contiene el Código de Aguas actualmente vigente, en el que se mantuvo sin alteraciones sustanciales

$47 \quad$ ROJAS (2010) p. 191. 
la normativa sobre organizaciones de usuarios y en particular sobre juntas de vigilancia establecida en el Código de Aguas de 1969, que, a su vez, en nada había alterado la del Código de 1951.

La principal diferencia que cabe destacarse es que los Códigos de Agua anteriores trataban las asociaciones de canalistas, aplicándose, en general, las normas de éstas a las comunidades de aguas y a las juntas de vigilancia. Por la inversa, el Código de Aguas vigente reglamenta detalladamente las comunidades de aguas, haciendo aplicable, en gran medida, las normas de éstas a las asociaciones de canalistas y, en conjunto, ambas se aplican a las juntas de vigilancia.

Reseñada la evolución de la normativa de aguas y en relación a las organizaciones de usuarios, nuestra regulación actual reconoce cuatro tipos, a saber: comunidades de aguas (superficiales y subterráneas); comunidades de drenaje; asociaciones de canalistas; $y$, juntas de vigilancia, todas las cuales comparten una serie de características comunes. Sin embargo, las disposiciones de estas organizaciones se han estructurado en función de las comunidades de aguas, quedando el resto relegadas a un segundo plano normativo. Cabe señalar que, en el contexto en que se originaron las organizaciones de usuarios, existía una mayor disponibilidad del recurso y, como consecuencia de ello, menor conflictividad. Asimismo, los usos que se le daba al agua eran principalmente el riego y, en menor medida, el consumo humano.

En la actualidad, el supuesto de hecho de las organizaciones de usuarios ha cambiado sustancialmente. Por una parte, como se señaló precedentemente, enfrentamos una disminución progresiva de la disponibilidad del recurso hídrico, lo que conlleva una mayor conflictividad entre los usuarios; y por la otra, con el desarrollo de la tecnología y la industria se incorporaron a la demanda de agua gran cantidad de nuevos y masivos usos, en algunos casos incompatibles entre sí, pero todos ellos esenciales para el desarrollo social y económico del país, como la generación hidroeléctrica y la gran minería, entre otros. No obstante los cambios en la situación de hecho descrita, el marco jurídico de las organizaciones de usuarios de aguas no ha sufrido cambios sustanciales, ya que en la actualidad sigue destinado principalmente a regular el riego, y si bien existen menciones a otros usos de aguas, distan mucho de ser herramientas útiles en la solución de los conflictos actuales y aquellos que se avecinan.

Por otra parte, actualmente tenemos la posibilidad de comprender de mejor manera las diversas interacciones existentes en una hoya hidrográfica: no solo ha aumentado la demanda y disminuido la disponibilidad del recurso hídrico, sino que también se ha tomado conciencia de su valor ambiental, 
por lo que cada vez cobra más importancia contar con un actor relevante en materia de gestión de los recursos hídricos a nivel de cuenca. Si bien nuestra legislación contempla una categoría superior de organizaciones de usuarios, las juntas de vigilancia, la posición que éstas ocupan en nuestra legislación genera una serie de dificultades que les impiden asumir un rol preponderante en la gestión de recursos hídricos, ya que se encuentran principalmente reguladas por las normas de las comunidades de aguas y asociaciones de canalistas, que solo tienen por objeto distribuir y administrar aguas en un cauce artificial y principalmente orientadas al riego. Ante un nuevo escenario, con menor disponibilidad de recursos hídricos, toma mayor relevancia el contar con instrumentos jurídicos que permitan conjugar las relaciones que se producen entre los diversos interesados en utilizar las aguas que les son comunes a nivel de cuencas hidrográficas. Dada su jurisdicción y potestades, las juntas de vigilancia constituyen el organismo más idóneo para asumir este rol, ya que son únicas como categoría de organizaciones de usuarios que administran las aguas desde sus fuentes naturales, y que son conformadas no solo por los usuarios individuales que extraen aguas desde el cauce, sino que por todos los titulares de derechos de aprovechamiento de aguas de una misma cuenca u hoya hidrográfica, sin posibilidad de exclusión.

\section{Reforma en trámite del Código de Aguas}

Actualmente la discusión que tiene por objeto modificar el Código de Aguas vigente ha radicado única y exclusivamente en lo referente a la propiedad de las aguas, su calidad de bien nacional de uso público y su preferencia para el consumo humano, quedando fuera cualquier argumento relativo a la gobernanza de las aguas.

En efecto, se ha presentado por parte del Gobierno actual una iniciativa por reformar el Código de Aguas, modificación que actualmente se encuentra en trámite ${ }^{48}$, cuyas propuestas más importantes pueden sintetizarse del siguiente modo: (i) otorgamiento de derechos de aprovechamiento por un período de tiempo determinado (máximo 30 años, prorrogable), no a perpetuidad, con la posibilidad además que éstos caduquen en caso que las aguas no sean efectivamente utilizadas. En este aspecto, se siguen modelos comparados como España y Argentina, cuyas legislaciones varían entre 3 y 5 años para dar un uso efectivo a las aguas antes de declarar su caducidad; (ii) dar facultades a la Administración del Estado para limitar el ejercicio de

48 "Proyecto de Ley que Reforma el Código de Aguas". Boletín n 7543-12, 8 septiembre 2014. Disponible en: <https://www.camara.cl/pley/pley_detalle.aspx?prmID=7936>, fecha de consulta: 5 de mayo de 2017. 
los derechos de aprovechamiento en épocas de escasez. Esta medida tiene por objeto hacerse cargo de la situación actual en que existe: (a) un sobre otorgamiento de derechos de aprovechamiento en cuencas agotadas; (b) así también una sobre explotación de los acuíferos que se explica por el mismo sobre otorgamiento, por las extracciones ilegales y por la falta de atribuciones para reducir de oficio ciertas extracciones; $y$, (c) finalmente un acaparamiento ocioso y especulación de derechos de aprovechamiento otorgados en forma gratuita. ${ }^{49}$ Así, se pretende mejorar la gestión del recurso hídrico en las cuencas que presentan estos problemas; (iii) establecer el consumo humano y saneamiento como uso prioritario, por sobre otros usos como, por ejemplo, el uso agrícola. Con esto se intenta solucionar el escaso acceso del mundo rural al agua, toda vez que no tienen el poder adquisitivo para adquirir derechos de aprovechamiento en el denominado "mercado de las aguas", posibilidad que sí tienen las empresas agro-exportadoras, mineras, forestales, eléctricas e industriales. De esta forma se pretende reconocer y concretar el derecho humano de acceder al agua potable y su saneamiento, para ser consistente con la Declaración Universal de los Derechos de la Humanidad ${ }^{50}$; (iv) para asegurar dicho uso -consumo humano y saneamiento- se establecerán reservas de aguas superficiales y subterráneas; (v) fortalecimiento de la Dirección General de Aguas. Esto ya que según la propia Dirección General de Aguas, Dirección del Ministerio de Obras Públicas, es un servicio que tiene escasas atribuciones e ineficiente intervención en fiscalización y aplicación de sanciones ante infracciones del Código de Aguas. Además, no existe en Chile una institucionalidad superior de los recursos hídricos; (vi) se prohíbe el otorgamiento de derechos de aprovechamiento en Parques Nacionales y Reservas de Región Virgen. Con esto se intenta resguardar el patrimonio ambiental, toda vez que el otorgamiento de derechos de aprovechamiento en Parques Nacionales y otras unidades protegidas ha permitido el desarrollo de proyectos hidroeléctricos al interior de estas áreas bajo protección oficial ${ }^{51}$; (vii)

49 Dirección General de Aguas y Ministerio de Obras Públicas (2014) p. 2.

50 Ibídem.

51 Al respecto, y si bien las áreas protegidas escapan al alcance del presente trabajo, se puede señalar que lo que se intenta con esa modificación es evitar que ante el vacío regulatorio existente se dicten fallos como, por ejemplo, aquel dictado por la Corte Suprema, Cisternas contra Comisión Regional del Medio Ambiente de la Región de Los Lagos (2009, rol n 6397-2008), sobre recurso de protección presentado en contra de la autoridad ambiental respectiva, por haber aprobado un proyecto hidroeléctrico al interior de un Parque Nacional, sentencia en la que concluyó que: "se puede concluir que es posible realizar proyectos u obras en parques nacionales y otras áreas protegidas. Lo exigido, conforme al marco jurídico que rige a esas unidades o áreas, es que la calificación favorable de tales proyectos sea precedida de una rigurosa evaluación de parte de la autoridad ambiental, 
se dan facilidades para que se otorguen derechos de aprovechamiento a los comités de agua potable rural, y asimismo se les exime del pago de la patente por no uso; (viii) se establece la obligación de inscribir los derechos de aprovechamiento y de regularizar los usos consuetudinarios en un plazo de 6 meses y de 1 año, respectivamente, contado desde la entrada en vigencia de la reforma.

No obstante lo anterior, cabe tener en consideración que solo se trata de un Proyecto de Ley, que aún no ha sido aprobado. Como se observa, se trata de medidas que tienen como objetivo central asegurar el consumo humano y declarar que las aguas son un bien nacional de uso público en cualquiera de sus estados, incluso una vez asignados los derechos a los particulares.

Actualmente, gran parte de la discusión respecto del Proyecto de Ley radica en la aplicación retroactiva de dichas disposiciones o si solo aplicarán para derechos constituidos a partir de su entrada en vigencia. La insistencia en uno u otro punto ha ido polarizando cada vez más las posiciones en contra o a favor del Proyecto de Ley. Sin embargo, el Proyecto de Ley en trámite nada dice respecto de la gobernanza del recurso hídrico, y en nuestra opinión, sea que la conclusión del Proyecto sea eliminar la propiedad particular sobre las aguas o mantenerlas, si no existe un buen sistema de gobernanza sobre las mismas, los efectos de la sequía y la sobreexplotación continuarán acrecentándose.

Por lo anterior, de acuerdo a lo expuesto, las discusiones y debates que se están dando actualmente en Chile en relación a la Reforma del Código de Aguas, lamentablemente, se han enfocado única y exclusivamente en la propiedad de las aguas, omitiendo la gobernanza de las aguas y el rol y responsabilidades que tendrá la Dirección General de Aguas en el futuro.

a fin que solo pueda ejecutarse bajo ciertas condiciones y exigencias en defensa del uso racional de los recursos naturales existentes. En otras palabras, por lo que se debe velar es que las actividades que se realicen dentro de los parques nacionales sean compatibles con los objetivos de dichos espacios de preservación de las bellezas naturales y de la flora y fauna asociadas". Asimismo, la prohibición de otorgar derechos en Parques Nacionales y Reservas de Región Virgen estaría acorde con lo dispuesto en el inciso final del artículo $3^{\circ}$ de la Convención para la Protección de la Flora, Fauna y Bellezas Escénicas de América, comúnmente denominada "Convención de Washington". Dicha norma señala que: "Los gobiernos contratantes convienen en que los límites de los parques nacionales no serán alterados ni enajenada parte alguna de ellos sino por acción de la autoridad legislativa competente. Las riquezas existentes en ellos no se explotarán con fines comerciales". 


\section{ELEMENTOS DE GOBERNANZA EN EL DERECHO DE AGUAS CHILENO}

La gobernanza del agua, según se ha entendido en este trabajo, debe considerar una interacción entre el Estado y actores no estatales en la toma de decisiones, para lograr un mecanismo de manejo y gestión del agua que satisfaga las necesidades de consumo de los diferentes actores interesados, con el objeto de diseñar políticas públicas y un marco regulatorio que no provoque la resistencia ni la obstaculización de los destinatarios de las normas. Es decir, un elemento esencial de la gobernanza es la participación de los diversos actores interesados en la toma de decisiones en el manejo de las aguas.

Cabe destacar que el ordenamiento jurídico de aguas chileno tuvo su origen en la necesidad de establecer reglas para el uso común de las aguas, por lo que sin haberse manifestado expresamente, desde sus inicios, la gobernanza de las aguas ha estado presente en nuestro ordenamiento jurídico configurado y manifestado en las organizaciones de usuarios de aguas.

Ahora bien, las disposiciones normativas referentes a dichas organizaciones, han tenido pocas variaciones desde su entrada en vigencia, a principios del Siglo $\mathrm{XX}$, por lo que resulta imperativo introducir actualizaciones a dicha estructura. Sin embargo, en las discusiones actuales no se han incorporado elementos de gobernanza. En esta línea, un aspecto que se observó en la investigación es que las organizaciones de usuarios tienen un rol fundamental en la gestión del recurso hídrico, en la medida que respondan a una integración participativa.

Para Hodgson ${ }^{52}$, muchos países han traspasado las tareas de gestión de las aguas desde agencias estatales hacia organizaciones de usuarios participativas, anónimas y autofinanciadas, lo que responde a que las agencias estatales no pueden afrontar ni operar de manera eficaz los sistemas de riego, de manera tal que las responsabilidades han sido transferidas a estas organizaciones de usuarios de aguas en un proceso comúnmente conocido como la "Transferencia de la Gestión del Riego" (Irrigation Management Transfer).

En Chile las organizaciones son consideradas personas jurídicas, integradas por agentes privados, que deben ser titulares de derechos de aprovechamientos que, en materia de riego, son en su mayoría agricultores. El impacto que tienen las organizaciones de usuarios de aguas en la producción agrícola en Chile es indudable, y ha sido tratado por McCarthy y Essam ${ }^{53}$, quienes concluyen que el análisis empírico revela que hay una relación entre

\footnotetext{
HOdGSON (2003) p. 1.

MCCARTHY y ESSAM (2009).
} 
la participación en las decisiones adoptadas por la organización, los montos de los aportes de sus miembros y los ingresos posteriores del sector agrícola.

Sin embargo, como se explicó, las organizaciones de usuarios en Chile están integradas por titulares de derechos de aprovechamiento, por lo que solo está presente una valoración del agua en el manejo de estas organizaciones de usuarios: el valor patrimonial económico del agua, extrañándose otras percepciones y valoraciones provenientes de otros actores, como quienes dan valor al disfrute del paisaje o quienes valoran el agua como un elemento esencial para la conservación del ecosistema. Es decir, en Chile las organizaciones de usuarios distan de ser participativas, sino que ellas son dirigidas por los intereses de unos pocos que deben ser titulares de derechos de aprovechamiento y que no necesariamente son actores locales ni habitantes del sector, puesto que, como se ha explicado, la legislación chilena no liga los derechos de aprovechamiento a la tierra.

En otras palabras, en Chile quien posee un derecho de aprovechamiento de aguas, no siempre tiene una merced de tierra disponible en la que utilice dichas aguas, es más, dicho titular puede-incluso- decidir no utilizar las aguas. En Alemania, por ejemplo, las organizaciones de usuarios, denominadas Wasser und Bodenverbände, son una organización tradicional de Derecho Público ${ }^{54}$ que comprende el manejo conjunto del agua y de la tierra. Las funciones de estas organizaciones son de interés público y están relacionadas, entre otras, con la administración de los cursos de aguas; construcción, mantención y operación de infraestructura, tal como obras de conducción de aguas y sistemas de regadío, drenaje y de defensa ante crecidas; y, ejecución de instalaciones que permiten el desarrollo de la actividad agrícola. Cada organización tiene su propio estatuto que debe ajustarse a las disposiciones legales. ${ }^{55} \mathrm{Es}$ un modelo de gobernanza que

54 Hodgson (2003) p. 8, plantea que en Alemania se agrega la expresión Verbander para indicar en forma expresa que estas organizaciones se rigen por el Derecho Público y así se les distingue de las asociaciones privadas regidas por el Código Civil de ese país.

55 La regulación de estas organizaciones está contenida en la Gesetz über Wasser und Bodenverbände (Ley de Asociaciones de Agua y Suelo de 1991). Esta ley prescribe las principales características de estas asociaciones, como su formación, tareas, composición (miembros), estructura interna, contenido de sus estatutos, financiamiento, entre otros. Además, en las diferentes regiones se han promulgado actos que permiten implementar lo establecido en la ley, los cuales son Ilamados Laenderausfuehrungsgesetze zum Geset züberWasserundBodenverbände. 
pretende aunar los intereses de los usuarios, disminuir los conflictos y que existan ganancias mutuas. ${ }^{56}$

Dicho lo anterior, resulta llamativa la diferencia en el tratamiento que se da en Chile y en Alemania respecto a las organizaciones de usuarios, en donde en unas -Chile- solo se gestionan las aguas, mientras que en las otras -Alemania- se hace un manejo del recurso hídrico que incorpora la tierra en la que se utilizan. Esto sin lugar a dudas puede ser materia de un trabajo de investigación comparativo que escapa al presente trabajo, pero que puede ser materia de una próxima investigación.

A partir de lo anterior, hace total sentido lo planteado por Nelson al indicar que: "La productividad agropecuaria, el acceso al mercado y los efectos del clima son en gran medida específicos a determinados lugares. Las agencias internacionales de desarrollo y los gobiernos nacionales deberían esforzarse para asegurar que el apoyo técnico, financiero y de fortalecimiento de capacidades llegue hasta las comunidades locales. También se debería alentar la participación comunal en los procesos nacionales de planificación de la adaptación. Las estrategias comunitarias de adaptación pueden ayudar a las comunidades rurales a reforzar su capacidad de sobrellevar desastres, mejorar sus habilidades de administración de tierras y diversificar sus medios de vida". ${ }^{57}$ De esta forma, es indudable que estas organizaciones de usuarios representan un actor importante en la gobernanza del agua y cumplen un rol en la toma de decisiones a nivel local que es relevante a la hora de tener un proceso participativo que incorpore diferentes percepciones en el manejo de las aguas.

Como señala Vergara58, en Chile "ni el Estado (esto es, los órganos del Estado) ni el mercado (esto es, la autónoma decisión de los particulares), por sí solos, han logrado con éxito que los individuos mantengan, a largo plazo, un uso productivo y positivo de los sistemas de recursos naturales. El caso de

56 Para Monsees (2004) p. 13, el éxito de este modelo deriva de la combinación, en síntesis, de los siguientes elementos: (i) reglas claras y flexibles contenidas en los estatutos de la asociación; (ii) garantizar la autonomía de la participación de los actores locales siempre en conformidad a la ley; (iii) evitar decisiones individuales (free-riders) mediante el establecimiento de una afiliación obligatoria; (iv) un equilibrio entre los derechos de los miembros y las facultades de la directiva de la asociación; (v) la evaluación meticulosa de los beneficios y costos de los miembros; (vi) financiamiento holgado; (vii) tener respeto por las normas rurales tradicionales (deeproots in traditional rural norms).

57 NeLSON et al. (2009) p. IX.

58 Vergara (2012) p. 1. 
las aguas es paradigmático en esta dualidad de manejo, a la vez, público y privado". Por esta razón, cabe recordar lo señalado por Retamal ${ }^{59}$ en cuanto a que, para superar estas debilidades, enfrentar el cambio climático y recorrer un camino de sustentabilidad, se requiere realizar modificaciones estructurales al actual sistema de gestión de las aguas en Chile, renovando realmente el marco normativo, promoviendo la participación de los diferentes actores y tomando en cuenta sus percepciones, valoraciones y conciencia en la toma de decisiones. Solo así se habrá incorporado la gobernanza en la regulación de las aguas en Chile. Así, la forma de integración y mecanismos de participación al interior de las organizaciones de usuarios de aguas es un tema interesante para ser tratado en el marco de una próxima investigación en materia de gobernanza de aguas, a fin de que sea incorporado ya sea en una política pública futura o en una eventual modificación del Código de Aguas.

\section{CONCLUSIONES}

De acuerdo a lo expuesto a lo largo del presente trabajo, la crisis del agua corresponde a una problemática de gobernanza del agua, en tiempos donde es preocupante la condición de sequía provocada por el cambio climático y la sobreexplotación del recurso hídrico.

Desde sus inicios, la legislación de aguas en Chile ha considerado a las comunidades de aguas, las asociaciones de canalistas y las juntas de vigilancia como una solución al uso común de las aguas por parte de los usuarios, pero no obstante la diversidad de usos que existen actualmente, el marco jurídico de las organizaciones de usuarios no ha sufrido cambios sustanciales y la regulación de estas entidades sigue orientada principalmente al riego, por lo que requiere de modificaciones normativas que las conviertan en vehículos útiles para la solución de los conflictos actuales y los que se vislumbran en el futuro, dotando a la gestión de las aguas de un sistema capaz de conciliar los diversos intereses que confluyen en una misma cuenca u hoya hidrográfica.

De acuerdo a lo expuesto en este trabajo, para que exista una buena gobernanza es imperativo que se incorporen nuevos actores en la toma de decisiones en torno al manejo y la gestión de las aguas, de manera tal que en las organizaciones de usuarios no solo tenga cabida el valor patrimonial económico del agua, sino que también se considere a otro tipo de entidades, tales como empresas de ecoturismo u organizaciones sociales que habitan en un lugar determinado, que dan otras valoraciones y que tienen otras percepciones igualmente válidas. Esto, por ejemplo, podría darse al interior de

$59 \quad$ Retamal et al. (2013) p. 15. 
CANNONI MANDUJANO, NICOLÁS Y CROCCO CARRERA, JUAN JOSÉ: GOBERNANZA Y DERECHO DE AGUAS EN CHILE

organizaciones de usuarios más participativas, que no solo incluyan a titulares de derechos de aprovechamiento.

\section{BIBLIOGRAFÍA CITADA}

Cámara de Diputados (1936): Sesión 11 a Ordinaria, 10 de junio de 1936

(Boletín de sesiones, Tomo I).

Daniell, Katherine A. y Barreteau, Olivier (2014): "Water governance across competing scales: Coupling land and water management", Journal of Hydrology, vol. 519, pp. 2367-2380.

Dirección General de Aguas y Ministerio de Obras Públicas (2014): Minuta de Reforma al Código de Aguas, 20 de agosto de 2014 (Santiago, Ministerio de Obras Públicas).

Donoso, Guillermo, Cancino, Joaé, Melo, Óscar, Rodríguez, Cristián y Contreras, Hugo (2010): Informe Final Análisis del Mercado del Agua de Riego en Chile: Una Revisión Crítica a través del caso de la Región de Valparaíso (Santiago, Pontificia Universidad Católica de Chile, Departamento de Economía Agraria).

Hodgson, Stephen (2003): Legislation on water users's organizations. A comparative analysis (Roma, Food and Agriculture Organization of the United Nations).

Marntz, Renate (1998): "Nuevos desafíos de la teoría de Governance (Trad. María Ángela Petrizzo)", Jean Monet Chair Paper RSC, n 98/50. Disponible en: <http://www.iue.it/RSC/Mayntz.htm>. Fecha de consulta: 5 de mayo 2017.

MCCARTHY, Nancy y ESSAM, Timothy (2009): Impact of Water User Associations on Agricultural Productivity in Chile (Washington, D.C. International Food Policy Research Institute, IFPRI).

MOnSEES, Jan (2004): "The German Water and Soil Associations - self-governance for small and medium scale water and land resources management", Working Paper on Management in Environmental Planning $\mathrm{n}^{\mathrm{o}}$ 10/2004.

NANDA, Ved (2006): "The Good Governance Concept Revisited", Annals of the American Academy of Political and Social Science, vol. 603, pp. 269-283. 
Nelson, Gerald, Rosegrant, Mark, Koo, Jawoo, Robertson, Richard, Sulser, Timothy, Zhu, Tinguv, Claudia, Msangi, Siwa, Palazzo, Amanda, Batka, Miroslav, Magalhaes, Marilia, Valmonte-Santos, Rowena, Ewing, Mandy y LeE, David (2009): Cambio Climático. El impacto en la agricultura y los costos de adaptación (Washington, D.C., Instituto Internacional de Investigación sobre Políticas Alimentarias IFPRI).

OBAndo, Iván (2009): "Estructura y jurisdicción de las organizaciones de usuarios de aguas en Chile durante el siglo XIX", Revista de Derecho de la Pontificia Universidad Católica de Valparaíso, vol. XXXII, pp. 107-132.

Organización para la Cooperación y el Desarrollo Económicos (2011): "Watergovernance in OECD countries: A multi-level approach. The water crisis is largely a governance crisis (Executive Summary)". Disponible en: <http://www.oecd.org/gov/regional-policy/48918283.pdf>, fecha de consulta: 21 de junio 2015.

(2015): OECD Principles on Water Governance. Welcomed by Ministers at the OECD. Ministerial Council Meeting on 4 June 2015 (Château de la Muette, Directorate for Public Governance and Territorial Development).

Retamal, Rafaela, Andreolı, Andrea, Arumi, José, Rojas, Jorge y Parra, Óscar (2013): "Gobernanza del agua y cambio climático: fortalezas y debilidades del actual sistema de gestión del agua en Chile. Análisis interno", Revista Interciencia, vol. $38 \mathrm{n}^{\circ} 1$, pp. 8-16.

Rojas, Christian (2010): La distribución de las aguas en el Derecho chileno. La actividad desarrollada por las juntas de vigilancia. Tesis para la obtención del grado académico de Doctor en Derecho (Santiago, Pontificia Universidad Católica de Chile).

Rosas-Ferrusca, Francisco, Calderón-Maya, Juan y Campos-Alanís, Héctor (2012): "Elementos conceptuales para el análisis de la gobernanza territorial", Quivera, vol. 14 n², pp. 113-136.

RuIz, Sergio y Gentes, Ingo (2008): "Retos y perspectivas de la gobernanza del agua y gestión integral de recursos hídricos en Bolivia", European Review of Latin American and Caribbean Studies, vol. 85, pp. 41-59.

Saldívar, Américo (2013): "Gobernanza multidimensional del agua: la Directiva Marco del Agua europea. Dificultades de su aplicación", Economía Informa, vol. 381, pp. 74-90. 
CANNONI MANDUJANO, NICOLÁS Y CROCCO CARRERA, JUAN JOSÉ: GOBERNANZA Y DERECHO DE AGUAS EN CHILE

Segura, Francisco (2009): Derecho de Aguas (Santiago, Legal Publishing).

StOKen, Gerry (1998): "Governance as theory: five propositions", ISSJ 155/1998, pp. 17-28.

Valdés-Pineda, Rodrigo, Pizarro, Roberto, García-Chevesich, Pablo, Valdés, Juan, Olivares, Claudio, Vera, Mauricio, Balocchi, Francisco, Pérez, Felipe, Vallejos, Carlos, Fuentes, Roberto, Abarza, Alejandra y Helwig, Bridget (2014): "Water governance in Chile: Availability, management and climate change", Journal of Hydrology, vol. 519, pp. 2538-2567.

VenEGAS, Fortunato (1887): Legislación Chilena sobre aguas de regadío (Santiago, Impr. i Lit. de la Seccion Técnica del E.M.J.).

Vergara, Alejandro (2012): "Focalizando la agenda de un recurso común: el desafío de potenciar la autogestión de las aguas en Chile", Centro de Políticas Públicas UC, año 7 n 56, pp. 1-11.

Vergara, Alejandro, Donoso, Guillermo, Rivera, Daniela, Blanco, Elisa y Morano, Valeria (2013): "Aguas y Energía: propuestas para su autogobierno y resolución especializada de conflictos", Concurso Políticas Públicas /2013, Propuestas para Chile, Capítulo VIII, pp. 241-270.

\section{NORMAS JURÍDICAS CITADAS}

Constitución Política de la República de Chile de 11 de agosto de 1980.

Código Civil de 1 de enero de 1857.

Código de Aguas del 29 de octubre de 1981, establecido por Decreto con Fuerza de Ley $n^{\circ} 1.122$.

Código de Minería de 14 de octubre de 1983, establecido por la Ley nº 18.248

Código de Procedimiento Civil de 30 de agosto de 1902, establecido por la Ley $\mathrm{n}^{\circ} 1.552$.

Decreto $\mathrm{n}^{\circ} 531$ del 4 de octubre de 1967, del Ministerio de Relaciones Exteriores, establece la Convención para la Protección de la Flora, Fauna y las Bellezas Escénicas Naturales de América.

Decreto Ley no 1552 de 13 de septiembre de 1976, modifica la Constitución Política de 1925, por medio de Acta Constitucional № 3. 
Decreto Ley no 2.603 de 23 de abril de 1979, modifica y complementa Acta Constitucional $\mathrm{N}^{\circ} 3$.

Ley de Asociaciones de Agua y Suelo de Alemania (Gesetz über Wasser und Bodenverbände) del 1 de mayo de 1991.

Ley $\mathrm{n}^{\circ} 2.139$ de 20 de noviembre de 1908, sobre asociaciones formadas por los dueños de los canales.

Ley $n^{\circ} 8.944$ de 11 de febrero de 1948, fija texto del Código de Aguas.

Ley no 9.909 de 28 de mayo de 1951, fija texto definitivo del Código de Aguas.

Ley nº 16.640 de 28 de julio de 1967, sobre Reforma Agraria.

Ley $n^{\circ} 20.017$ de 16 de junio de 2005, modifica el Código de Aguas.

Ordenanza de 3 enero de 1872, sobre distribución de las aguas en los ríos que dividen provincias y departamentos.

\section{JURISPRUDENCIA CITADA}

Corte Suprema, Cisternas contra Comisión Regional del Medio Ambiente de la Región de Los Lagos (Recurso de Protección) (2009): 8 enero 2009, rol n $6397-2008$. 